\title{
Atlas of Circulating Immune Cells in Kawasaki Disease
}

\author{
Zhenyu Xie ${ }^{1}$, Yinde Huang ${ }^{1}$, Xinyang $\mathrm{Li}^{1}$, Yu Lun ${ }^{1}$, Xin $\mathrm{Li}^{1}$, Yuzhen $\mathrm{He}^{1}$, Song $\mathrm{Wu}^{1}$, \\ Shiyue Wang ${ }^{1}$, Jianjian Sun ${ }^{1}$, and Jian Zhang ${ }^{1}$ \\ ${ }^{1}$ China Medical University Second Hospital
}

July 2, 2021

\begin{abstract}
Increased evidence shows that the pathogenesis of Kawasaki disease (KD) is caused by abnormal and unbalanced innate and adaptive immune responses. However, the changes in and functions of adaptive immune cells in KD peripheral blood are still controversial. In this study, three different methods, CIBERSORT, Immune Cell Abundance Identifier (ImmuCellAI), and immune cell markers, were used to evaluate the proportions and abundances of immune cells in eight KD datasets (GSE9863, GSE9864, GSE18606, GSE63881, GSE68004, GSE73461, GSE73463, and GSE64486; a total of 1,251 samples). Compared with those in normal controls and convalescent KD samples, the proportions and abundances of innate immune cells such as neutrophils, monocytes, and macrophages in acute KD peripheral blood samples were significantly increased, while those of adaptive immune cells such as B and $\mathrm{T}$ cells were significantly decreased. The change tendency of these immune cells was similar to that seen in other febrile illnesses but more dramatic. However, in the coronary artery tissue of KD patients, adaptive immune cells, especially B cells and CD8+ T cell subsets, were significantly increased. This result suggests that adaptive immune cells are selectively recruited from peripheral blood into the coronary arteries. In addition, we found that elevated neutrophils in peripheral blood could be used as a biomarker to assist in the differential diagnosis of KD, but we did not find immune cells that can accurately predict intravenous immunoglobulin (IVIG) response in multiple datasets.
\end{abstract}

\section{Introduction}

Kawasaki disease (KD) is an acute childhood vasculitis that mainly affects children from 6 months to 5 years old [1-3]. KD can cause coronary artery aneurysms (CAAs) and is the main cause of acquired heart disease in children in developed countries $[4,5]$. The cause is not yet clear; therefore, diagnosis and treatment are still based on experience, and KD cannot be prevented. High-dose intravenous immunoglobulin (IVIG) is the main method of treatment in the acute phase, reducing the risk of aneurysms from approximately $25 \%$ to $4 \%$. However, approximately $20 \%$ of patients do not respond to the initial treatment of IVIG, leading to increased risk of aneurysms [6].

$\mathrm{KD}$ is related to the infiltration of coronary artery walls by a variety of innate and adaptive immune cells $[5,7]$. Based on the study of postmortem tissue in KD patients, Orenstein et al. [8] divided the vascular pathological process of KD into three steps: necrotizing arteritis develops in the first two weeks of the disease and is related to neutrophil infiltration, which gradually destroys the intima, media, and portions of the adventitia of the coronary artery; subacute/chronic vasculitis is induced by inflammatory infiltrates composed of CD8+ $\mathrm{T}$ cells, IgA+ plasma cells, eosinophils and macrophages; and luminal myofibroblastic proliferation (LMP), which is a process in which modified medial smooth muscle cells proliferate and gradually block the arterial lumen.

The innate immune response plays an important role in KD systemic and vascular inflammation. It is generally believed that the acute phase response of KD is mainly driven by the innate immune system [911]. Neutrophils, monocytes, and macrophages in the peripheral blood of acute KD are also significantly increased [12-17]. Recent studies have gradually realized that acquired immunity also plays an important 
role in $\mathrm{KD}[10,18]$. The decrease in the proportions and numbers of lymphocytes in the peripheral blood of KD has been well recognized $[12,15,19]$. However, the changes in and functions of adaptive immune cells, especially B cells and CD4+ T cell subsets in peripheral blood, are still controversial $[12,13,17,20-33]$. In addition, research on $\mathrm{T}$ cells focuses on CD4+ T cells, CD8 $+\mathrm{T}$ cells, Tregs, and Th17 cells, while other $\mathrm{T}$ cell subsets still lack relevant reports in KD.

Based on bioinformatics methods, we established a KD peripheral immune cell atlas and focused on the following two main questions.

1. How do the proportions and numbers of immune cells in peripheral blood change during acute and convalescent KD? 2. Can peripheral blood immune cells be used to diagnose KD or predict IVIG response?

The former is a basic research question that prompts exploration of the immune characteristics of KD, which will aid the understanding of the pathogenesis of $\mathrm{KD}$, so that more targeted therapies can be used to replace IVIG; in contrast, the latter question prompts the identification of immune cells as biomarkers to improve timely treatment. These two questions are also the focus of current KD research.

\section{Methods}

\subsection{Materials}

Seven whole-blood RNA microarray datasets (GSE9863, GSE9864, GSE18606, GSE63881, GSE68004, GSE73461, and GSE73463) and one coronary artery high-throughput RNA sequencing dataset (GSE64486) were downloaded from the Gene Expression Omnibus (GEO) database (http://www.ncbi.nlm.nih.gov/geo) (Table 1).

\subsection{Screening of Differentially Expressed Genes (DEGs)}

GEO2R (http://www.ncbi.nlm.nih.gov/geo/geo2r/) was used to screen the DEGs between the normal controls and KD patients (adjusted p-value <0.05). We defined common DEGs in GSE18606, GSE68004, and GSE73461 as verified differentially expressed genes (VDEGs).

\subsection{Pathway and Process Enrichment Analysis}

Metascape [34] (http://metascape.org/) is a web-based portal for gene function annotation analysis. The top 20 clusters with their representative enriched terms were obtained using Metascape with all VDEGs as the enrichment background. Additionally, DisGeNET [35] (https://www.disgenet.org) was used to explore VDEG-associated human diseases.

\subsection{Gene Set Enrichment Analysis (GSEA)}

GSEA software [36] (https://www.gsea-msigdb.org/gsea/) (version 4.0.3) was used to explore the main biological functions that caused differences between the normal controls $(\mathrm{n}=101)$ and KD patients $(\mathrm{n}=173)$. The data were derived from GSE18606, GSE68004, and GSE73461 and cleaned by the "limma" and "SVA" packages in R. "h.all.v7.1.symbols.gmt" was used as the reference gene set with 1,000 permutations for each analysis. |normalized enrichment score (NES)| [?] 1.0,p -value [?] 0.05, and false discovery rate (FDR) q-val [?] 0.25 were used as thresholds for determining statistical significance.

\subsection{Calculation of the Proportions and Abundances of Immune Cells}

\subsubsection{Cibersort}

CIBERSORT [37] is a deconvolution algorithm based on RNA-seq data. It was used to calculate the proportions of 22 immune cells in each sample, and the sum was 1 . To ensure the reliability of the results, we performed 1,000 permutations and retained samples with $p<0.05$. In this study, CIBERSORT was used to calculate the proportions of immune cells in KD samples and control samples.

\subsubsection{Immune Cell Abundance Identifier (ImmuCellAI)}


ImmuCellAI [38] (http://bioinfo.life.hust.edu.cn/web/ImmuCellAI/) is a method based on single sample gene set enrichment analysis (ssGSEA) that can be used to accurately estimate the abundances of 24 immune cells from gene expression data, including $18 \mathrm{~T}$ cell subgroups. T cells are composed of two major groups: CD4+ T cell subsets, such as T helper cells [e.g., Th1, Th2, Th17, and T follicular helper (Tfh) cells] and regulatory $\mathrm{T}$ cells (e.g., iTreg, nTreg, and Tr1 cells); and CD8+ T cell subsets, such as cytotoxic T cells, exhausted T cells and mucosal-associated invariant T (MAIT) cells. In this study, ImmuCellAI was used to calculate the abundances of immune cells in KD samples and control samples.

Additionally, we also used immune cell markers to estimate the abundances of immune cells as a means of supplementary verification [39]. To facilitate visualization, each gene was normalized by $\log _{2}(\operatorname{expresion}+1)$ in particular datasets.

\subsection{Statistical Analysis}

The Mann-Whitney U test was used for comparisons between two groups. The Kruskal-Wallis test was used for comparisons between multiple groups. The statistical analysis of the data was performed by $\mathrm{R}$ (version 3.6.0). $p<0.05$ was considered to indicate statistical significance.

\section{Results}

3.1 The Inflammatory Response is the Most Significant Hallmark of KD

In GSE18606, GSE68004, and GSE73461, there were a total of 2,999 common DEGs between normal controls and acute KD samples, comprising 1,546 upregulated and 1,453 downregulated DEGs (Figure 1A). When these VDEGs were used as the enrichment background to conduct pathway and process enrichment analysis in Metascape, the results showed that immune-related pathways were the most frequently noted pathways. The top 20 significant enrichment clusters are shown in Figure 1B, among which "myeloid leukocyte activation", "leukocyte differentiation", "cellular response to stress", "activation of immune response", and "adaptive immune system" are the top five significant enrichment clusters. The Metascape enrichment network showed a high degree of similarity of immune-related enrichment terms within and between the top 20 clusters (Figure 1C). GSEA was also used to identify the main biological function differences between the normal controls $(\mathrm{n}=101)$ and acute KD samples ( $\mathrm{n}=173)$ of GSE18606, GSE68004, and GSE73461 (Figure 1D). The results showed that the inflammatory response is the most significant hallmark of KD. 
A

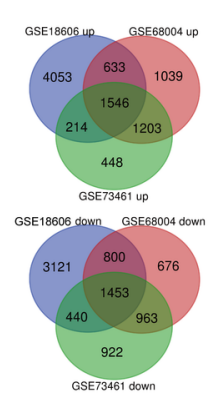

B

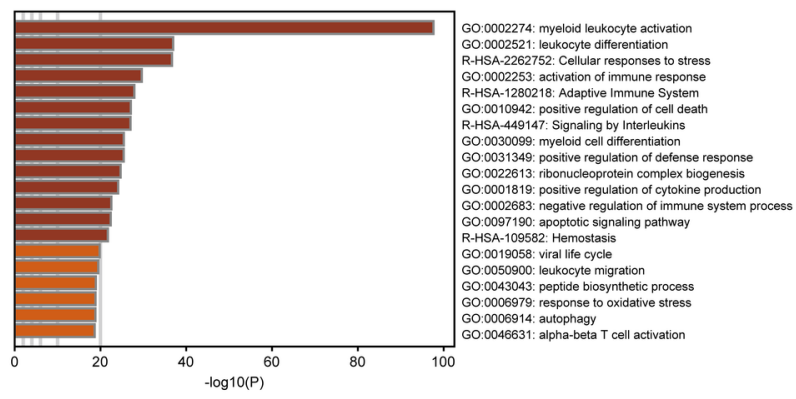

D
C

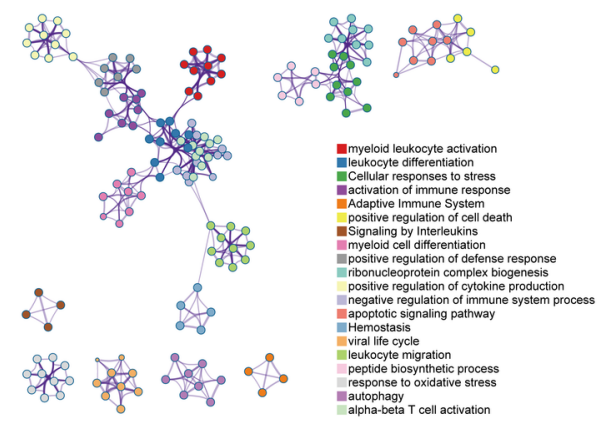

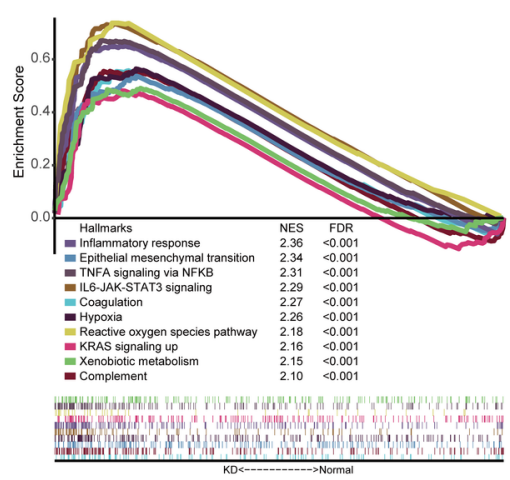

Additionally, "pneumonitis", "infection", and "bacterial infections" were the three most significant VDEGassociated human diseases identified via DisGeNET (Figure S1), which suggests that pathogens that infect through the respiratory tract could be important causes of KD.

In summary, the immune response is the most important feature of KD.

3.2 In Acute KD Peripheral Blood, Innate Immune Cells are Increased, While Adaptive Immune Cells are Decreased

To describe the dynamic changes in peripheral blood immune cells in KD, CIBERSORT was used to calculate the proportion of each of the 22 immune cell types based on KD microarray data. We separately determined the composition of peripheral blood immune cells in the normal control group ( $\mathrm{n}=101$, GSE18606, GSE68004, and GSE73461), acute KD group (n=480, GSE18606, GSE63881, GSE68004, GSE73461, and GSE73463) and convalescent KD group ( $\mathrm{n}=101$, GSE18606, GSE63881, and GSE73463) (Figure 2A-C). We found that the proportion of innate immune cells represented by neutrophils, monocytes, and macrophages was significantly increased and that of adaptive immune cells represented by $\mathrm{B}$ cells and $\mathrm{T}$ cell subsets was significantly decreased in acute KD (Figure 2D). In convalescent KD, the aforementioned changes in the proportion of immune cells were restored.

We determined the differences in immune cell proportions between normal and acute KD samples, and between acute KD and convalescent KD samples in GSE18606, GSE63881, GSE68004, GSE73461, and GSE73463 (Figure 2E and Figures S2 and S3). The results that show the same significant trend in the comparison of 4 or more datasets are considered to have high credibility. Using this as a standard, our results showed that compared with the respective values in normal and convalescent KD samples, the proportions of 
monocytes, M0 macrophages, and neutrophils in acute KD samples were significantly increased, while those of memory B cells, CD8+ T cells, resting CD4+ T cells, resting natural killer (NK) cells, activated NK cells, and M2 macrophages were significantly decreased.

A
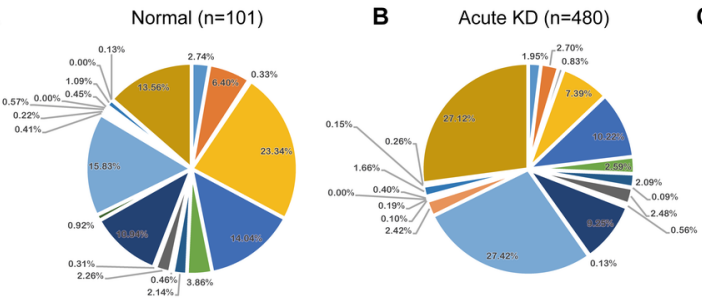

C Convalescent KD ( $\mathrm{n}=272)$
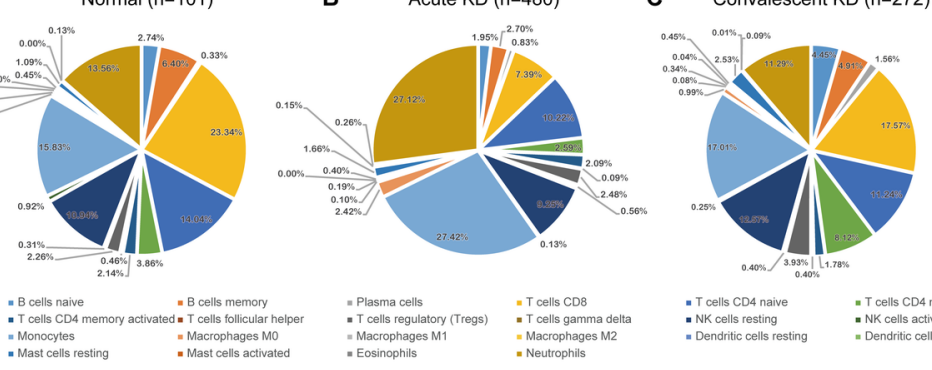

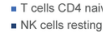

D
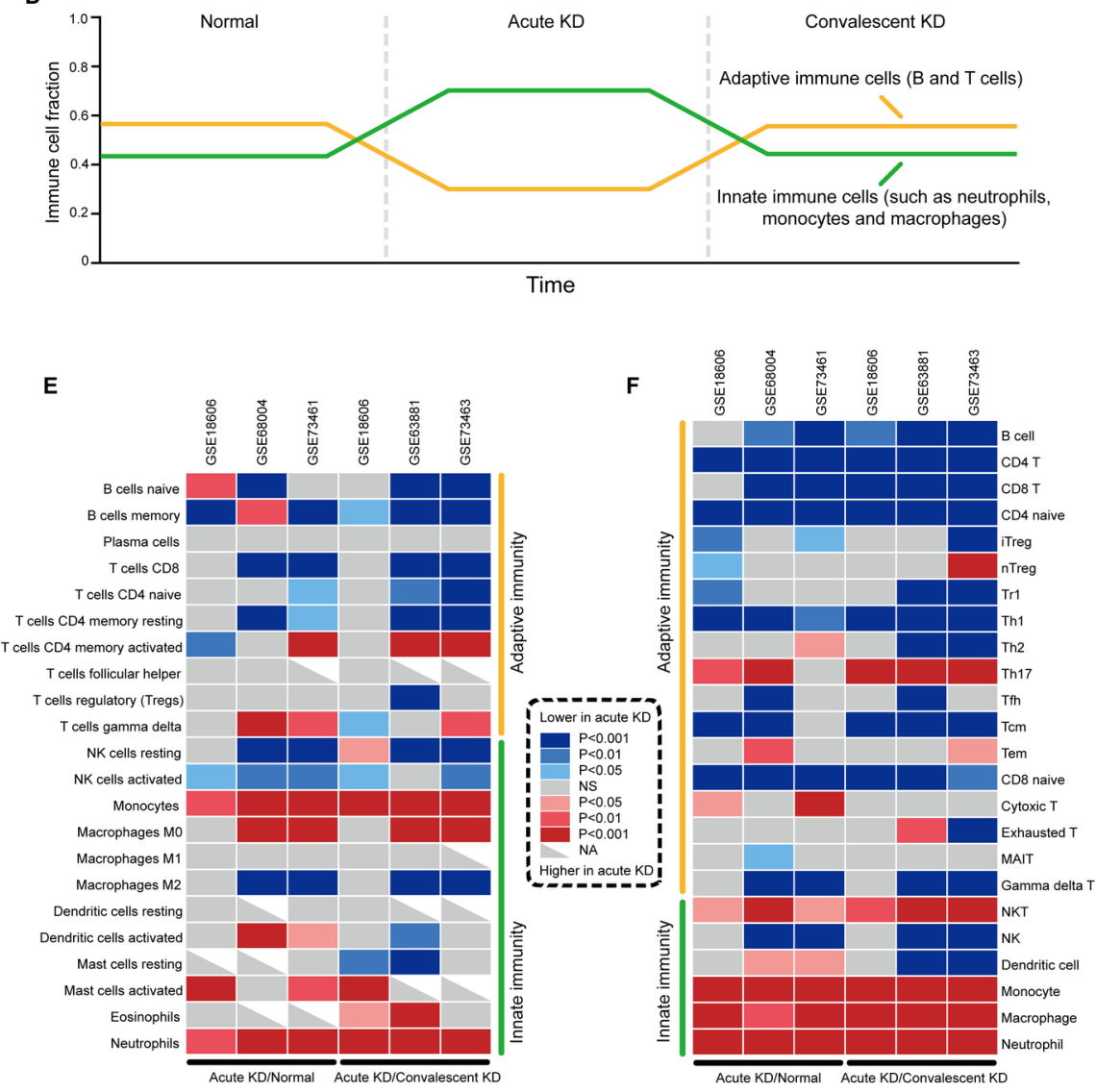

Similarly, we determined the differences in immune cell abundances (ImmuCellAI) (Figure 2F, Figures S4 and S5, and Tables S1 and S2). The results showed that compared to the respective values in normal and convalescent KD samples, the abundances of Th17 cells, natural killer T (NKT) cells, neutrophils, monocytes, and macrophages in acute KD samples were significantly increased, while those in B cells, CD4+ T cells, CD8+ T cells, CD4+ naive T cells, Th1 cells, central memory T $(\mathrm{Tcm})$ cells, naive CD8+ T cells, gamma delta $\mathrm{T}(\gamma \delta \mathrm{T})$ cells and NK cells were significantly decreased.

In addition, comparison of immune cell markers in GSE9863, GSE18606, GSE63881, GSE68004, GSE73461, 
and GSE73463 (Figures S6-S8) also showed that CD68 (a macrophage marker) and CXCR2 (a neutrophil marker) levels in acute KD samples were significantly higher than those in normal and convalescent KD samples, while MS4A1 (a B cell marker), CD8A (a cytotoxic T cell marker), CD4 (a T helper cell marker), CXCR5 (a Tfh marker), B3GAT1 (an NK cell marker) levels were significantly decreased.

In summary, compared with the respective values in normal controls and convalescent KD samples, the proportions and abundances of innate immune cells represented by neutrophils, monocytes, and macrophages were significantly increased while those of adaptive immune cells represented by B cells and $\mathrm{T}$ cell subsets were significantly decreased in acute KD peripheral blood samples.

3.3 The Changes in Peripheral Blood Immune Cells in Acute KD are Similar to those Derived from Febrile Controls but More Dramatic

To explore the differences between peripheral blood immune cells in KD and other febrile diseases, the febrile controls of GSE68004 and GSE73461 were also included in the study. The results showed that compared with those in the normal controls, the immune cell changes in the acute KD samples and febrile controls were similar. Both showed increased innate immune cells and decreased adaptive immune cells, but the degree of change in the acute KD samples was more dramatic (Table 2). In particular, the decrease in CD4+ T cells and CD8+ T cells and the increase in neutrophils in KD peripheral blood samples were specific compared to febrile controls (Table 2 and Figure S9). Peripheral blood neutrophil abundance performed well in the differential diagnosis of KD samples from normal controls and high fever controls (Figure S10). In addition, the InfiltrationScore, which represents the degree of immune response in ImmuCellAI, was also highest in acute KD (Table 2).

\subsection{There are no KD Peripheral Blood Immune Cells that can Accurately Predict the IVIG Response}

Generally, a patient with persistent or recurrent fever [?]36 h after the completion of the initial IVIG infusion is defined as an IVIG nonresponder [40]. In GSE63881, IVIG nonresponders accounted for a minority of the KD patients $(61 / 171)$, but they accounted for the majority of the patients with a diagnosis of coronary angioma (15/20) (Figure 3A). Therefore, we explored whether peripheral blood immune cells can be used to predict the IVIG response. We compared the proportions and abundances of immune cells between IVIG responders and IVIG nonresponders in acute KD and convalescent KD (Figure 3B-E and Figures S11-S13). However, no peripheral blood immune cells that could accurately predict the IVIG response in multiple datasets were identified. 
A

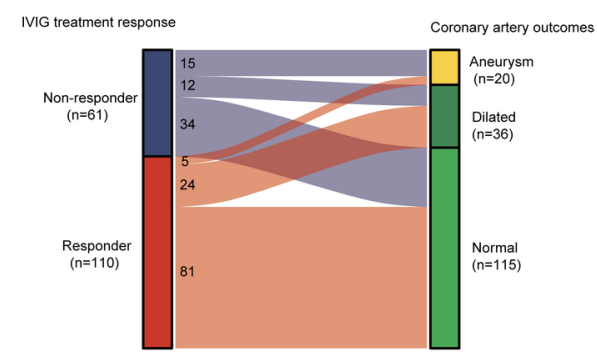

c

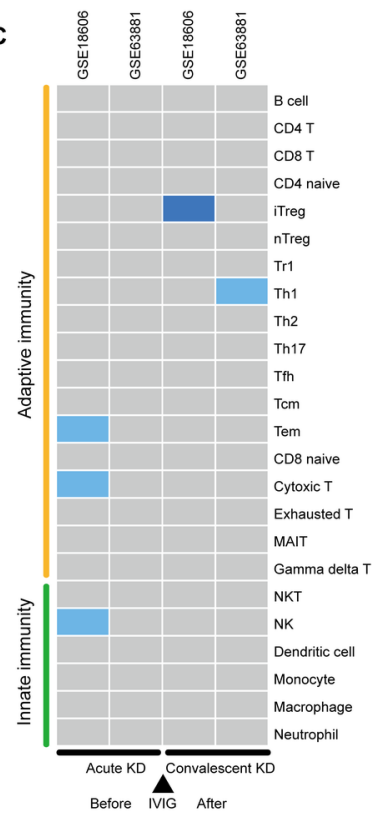

B
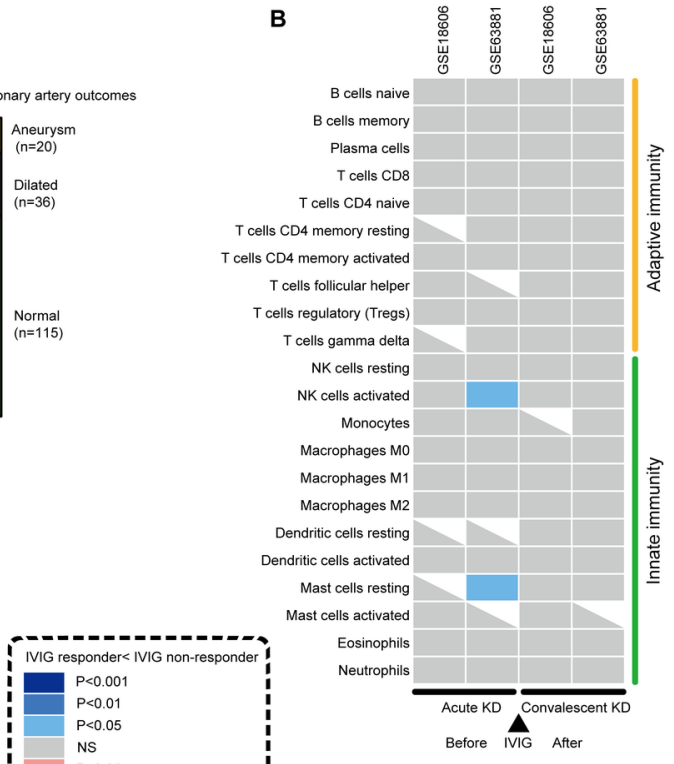

鹿

窟

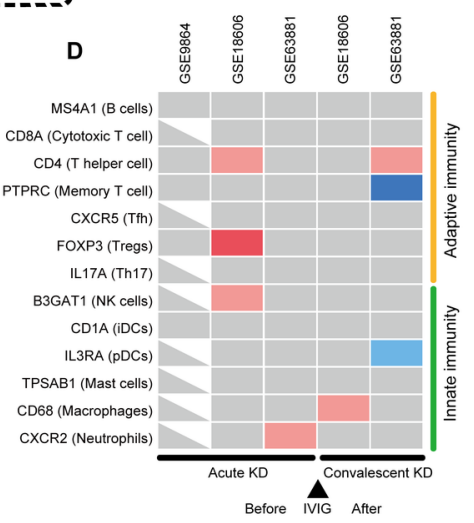

In addition, we also analyzed the relationship between KD peripheral blood immune cells and coronary aneurysm formation in GSE63881, and we did not find any immune cells that were highly related to aneurysm formation (Figure S14).

3.5 There is High Infiltration of Adaptive Immune Cells in KD Coronary Arteries 


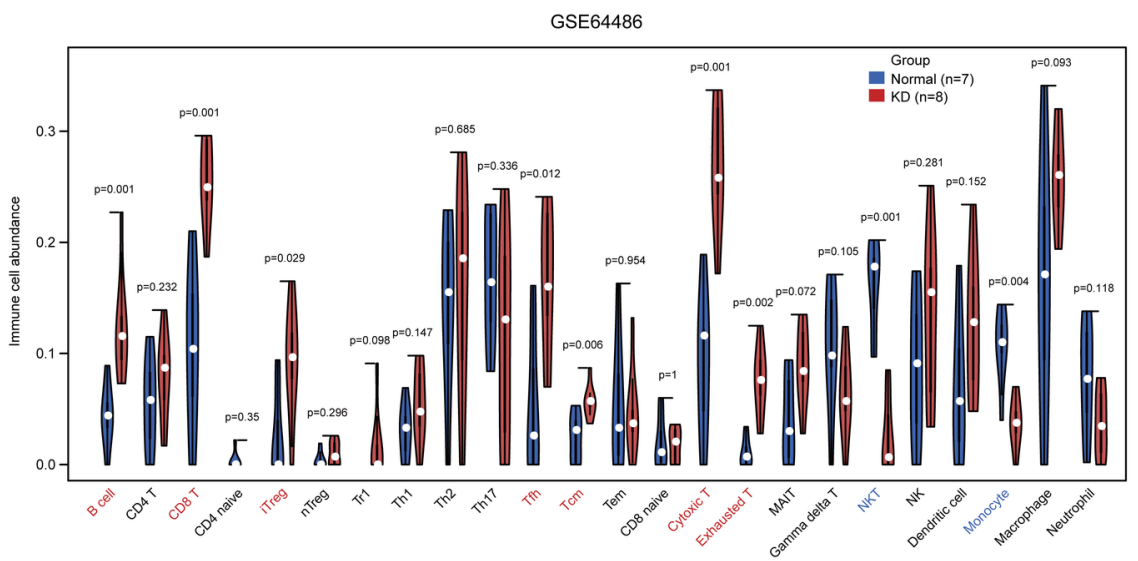

We further compared the difference in immune cell infiltration between KD coronary arteries and normal coronary arteries in GSE64486. The ImmuCellAI results showed that in KD coronary arteries, the abundances of B, CD8+ T, iTreg, Tfh, Tcm, cytotoxic T and exhausted $\mathrm{T}$ cells were significantly increased, while those of NKT cells and monocytes were significantly decreased (Figure 4). In addition, the results of immune cell biomarkers showed that almost all the markers of immune cells showed an upward trend, including MS4A1 (B cells), CD8A (cytotoxic T cells), CD4 (T helper cells), PTPRC (memory T cells), and CXCR2 (neutrophils), which reached a statistically significant level (Figure S15).

In summary, adaptive immune cells, especially B cells and CD8+ T cell subsets, are significantly increased in KD coronary arteries.

\section{Discussion}

In this study, by using three different algorithms to evaluate the proportions and abundances of immune cells in a total of 1,251 samples from eight GEO datasets, we established an atlas describing the changes in KD peripheral blood immune cells. The results show that compared with those in normal controls and convalescent KD samples, the proportions and abundances of innate immune cells such as neutrophils, monocytes, and macrophages in acute KD peripheral blood samples are significantly increased, while those of adaptive immune cells such as B and $\mathrm{T}$ cells are significantly reduced. The change tendency of these immune cells is similar to that seen in other febrile illnesses but more dramatic. In the coronary arteries of KD, adaptive immune cells, especially B cells and CD8+ T cell subsets, are significantly increased. This finding suggests that adaptive immune cells are selectively recruited from peripheral blood into the coronary arteries. In addition, we found that elevated neutrophils in peripheral blood could be used as a biomarker to assist in the differential diagnosis of $\mathrm{KD}$, but we did not find immune cells that could accurately predict IVIG response in multiple datasets. 


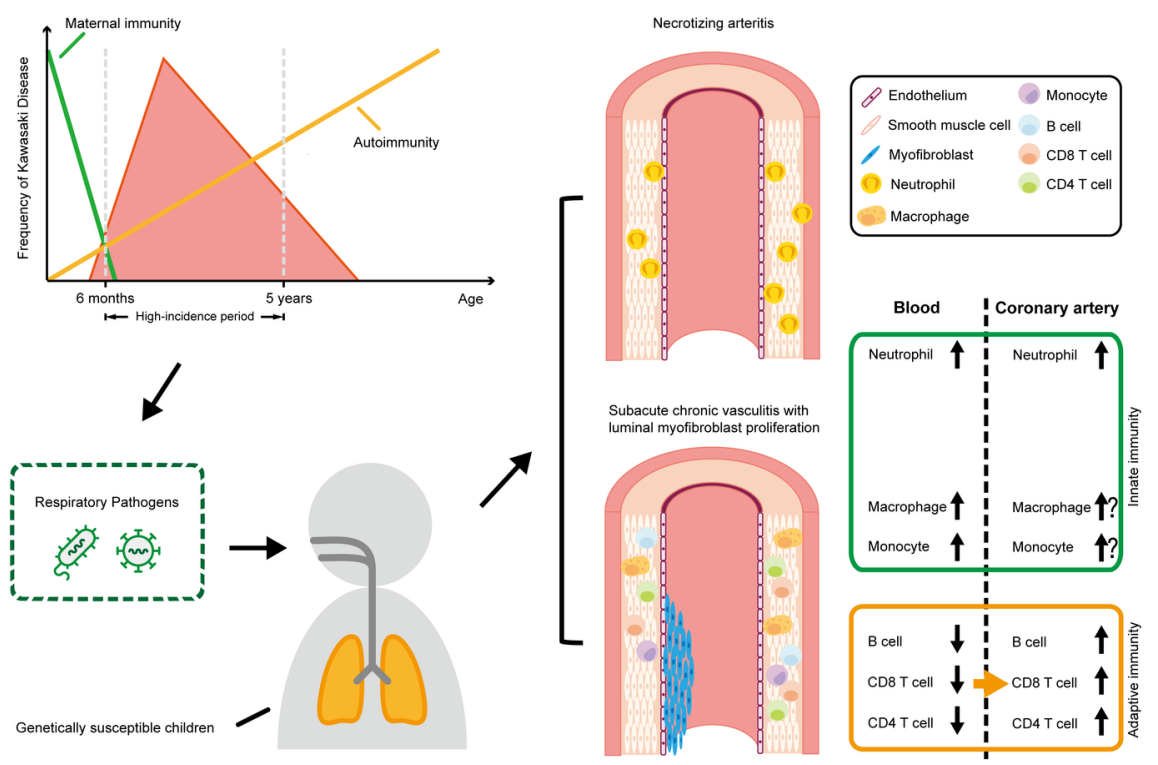

KD pathogenesis is currently understood to include a pathologically amplified immune response in children who are genetically susceptible to pathogens that are believed to be widely dispersed in the environment and to enter through the respiratory tract $[2,41,42]$ (Figure 5). Our results show that "pneumonitis", "infection", and "bacterial infections" are the three most significant VDEG-associated human diseases according to DisGeNET, which supports that respiratory tract infection is an important cause of KD. In addition, our research shows that the change trend of peripheral blood immune cells in acute KD is similar to that seen in febrile illnesses caused by bacterial or viral infection but is more dramatic, which also suggests that KD is an excessive immune response to common infectious agents. In addition, outbreaks of KD around the world during the COVID-19 pandemic have shown that the agents that induce KD are nonspecific $[43,44]$.

Increased evidence suggests that KD is caused by abnormal and imbalanced innate and adaptive immune responses $[18,45]$. Our functional and pathway enrichment analysis results also showed that the inflammatory response is the most significant hallmark of KD. At present, many studies have described the changes in KD peripheral blood immune cells (Table 3 ). The significant increase in innate immune cells in acute KD has been well recognized. However, the changes in and functions of adaptive immune cells, especially B cells and CD4+ T cell subsets, are still controversial. In recent years, research has suggested that B cells in the peripheral blood of acute KD are elevated [12,16,17,20-23,46-53]. However, our results show that B cells decrease significantly in acute KD and return to the levels in normal samples in convalescent KD. In addition, it is worth mentioning that our study is the first to use ImmuCellAI to calculate the abundances of $18 \mathrm{~T}$ cell subsets in KD peripheral blood, and the abundances of most $\mathrm{T}$ cell subsets have not been reported in KD. Our results showed that compared with the respective values in normal controls and convalescent KD samples, the abundances of CD4+ T cells, CD8+ T cells, CD4+ naive T cells, Th1 cells, Tcm cells, naive CD8+ T cells, and $\gamma \delta \mathrm{T}$ cells in acute KD samples were decreased significantly, while the abundances of Th17 cells and NKT cells were significantly increased. The detailed classification of T cells helps us to understand the features and functions of $\mathrm{T}$ cell subsets in KD more accurately.

$\mathrm{KD}$ is closely related to the infiltration of coronary artery walls by a broad variety of innate and adaptive immune cells $[5,7,9,72]$ Research on postmortem tissues of KD patients has indicated that neutrophils are the first responders to invade the arterial wall, followed by monocytes/macrophages, dendritic cells and CD8+ T cells $[7,8]$. These infiltrating immune cells release proinflammatory cytokines such as IL- $1 \beta$ and 
TNF to promote the development of vascular endothelial cell injury and CAA [7]. We also observed an increase in the abundances of adaptive immune cells in the coronary arteries of KD patients in GSE64486, with the increased abundances of B cells and CD8+ T cells being particularly significant. Combined with the significant decrease in adaptive immune cells seen in the peripheral blood of acute KD indicates that adaptive immune cells, especially B cells and CD8+ T cells, are selectively recruited into the coronary arteries in acute KD. This result also provides a theoretical basis for anti- $\mathrm{T}$ cell therapies, such as tacrolimus and the calcineurin inhibitor cyclosporine [73,74], which can quickly control inflammation and are expected to become an effective choice for refractory KD.

We also explored the clinical application value of immune cells in KD by looking for peripheral blood immune cells that can be used to diagnose KD or predict the response to IVIG. We found that the changes in acute KD peripheral blood immune cells were similar to those seen in febrile controls but more dramatic. Among the changes, the increase in neutrophils was specific for KD compared with other febrile illnesses, which indicates that neutrophils may be used as a biomarker to assist the diagnosis of KD. Clinically, IVIG reduces the prevalence of coronary artery abnormalities by reducing tissue inflammation and immune activation $[40,75]$. Patients with IVIG resistance are at higher risk of developing CAA, and other treatments are needed to control inflammation [76]. Therefore, finding biomarkers that can predict IVIG resistance is an important area of current KD research. Neutrophils [14,77-81], B cells [23], CD8+ T cells [82], and Th17 cells $[32,33]$ in KD peripheral blood have all been reported to predict IVIG resistance, but the types of immune cells supported by different studies are not consistent. Our research did not identify immune cells that can accurately predict IVIG response in multiple datasets. Therefore, the use of immune cell biomarkers to predict IVIG response still needs further exploration and more data verification.

In short, we used public databases to build an atlas that can comprehensively and accurately describe the dynamic changes in the proportions and abundances of KD peripheral blood immune cells. This research is undoubtedly an important basis for studying the immune mechanism of KD. In addition, the bioinformatics research template we use in this study is highly reproducible and can be widely used in immune research on other diseases (such as cancers) using peripheral blood or tissues.

However, this study has some limitations. First, this study is a descriptive study and did not involve the study of the specific biological functions and internal mechanisms of KD peripheral blood immune cells. Second, the coronary artery samples were all from autopsy samples, and the sample size was small, which may affect the accuracy of the results. For example, our study did not show that monocytes and macrophages infiltrate the KD coronary arteries, but they have been reported to do so in the literature, and the abundance of monocytes in this study was even significantly decreased. Fortunately, with the increasing amount of public data and relevant clinical information, the pathological changes and clinical value of immune cells in KD will be more deeply understood. In recent years, single-cell sequencing has developed rapidly, and major breakthroughs have been made in the study of the mechanism of other vascular diseases $[83,84]$. Such advances are expected to become an important piece in the field of KD immune research.

\section{Acknowledgements}

We would like to thank the contributions of public databases and data providers to human medicine.

\section{References}

1 McCrindle BW, Rowley AH, Newburger JW et al. Diagnosis, treatment, and long-term management of Kawasaki disease: a scientific statement for health professionals from the American Heart Association. Circulation 2017; 135 :e927-99.

2 Greco A, De Virgilio A, Rizzo MI et al. Kawasaki disease: an evolving paradigm. Autoimmun Rev 2015; $14: 703-9$.

3 Uehara R, Belay ED. Epidemiology of Kawasaki disease in Asia, Europe, and the United States. J Epidemiol 2012; 22 :79-85. 
4 Kato H, Koike S, Yamamoto M et al. Coronary aneurysms in infants and young children with acute febrile mucocutaneous lymph node syndrome. J Pediatr 1975; 86 :892-8.

5 Newburger JW, Takahashi M, Burns JC. Kawasaki disease. J Am Coll Cardiol 2016; 67 :1738-49.

6 McCrindle BW, Rowley AH. Improving coronary artery outcomes for children with Kawasaki disease. Lancet 2019; $393: 1077-8$.

7 Rivas MN, Arditi M. Kawasaki disease: pathophysiology and insights from mouse models. Nat Rev Rheumatol 2020; $16: 391-405$.

8 Orenstein JM, Shulman ST, Fox LM et al. Three linked vasculopathic processes characterize Kawasaki disease: a light and transmission electron microscopic study. PLoS One 2012;7 :e38998.

9 Lo MS. A framework for understanding Kawasaki disease pathogenesis. Clin Immunol 2020; 214 :108385.

10 Hedrich CM, Schnabel A, Hospach T. Kawasaki disease. Front Pediatr 2018; 6 :198.

11 Hara T, Nakashima Y, Sakai Y et al. Kawasaki disease: a matter of innate immunity. Clin Exp Immunol $2016 ; 186: 134-43$.

12 Zhang C, Zhang X, Shen J et al. Changes in peripheral blood neutrophils, lymphocytes and IL-10 in children with Kawasaki disease from different age groups undergoing intravenous immunoglobulin: a retrospective study. Mediators Inflamm 2020; 2020 :5213451.

13 Consiglio CR, Cotugno N, Sardh F et al. The immunology of multisystem inflammatory syndrome in children with COVID-19. Cell 2020;183:968-81.e7.

14 Tsujimoto H, Takeshita S, Nakatani K et al. Intravenous immunoglobulin therapy induces neutrophil apoptosis in Kawasaki disease. Clin Immunol 2002; $103: 161-8$.

15 Hoang LT, Shimizu C, Ling L et al. Global gene expression profiling identifies new therapeutic targets in acute Kawasaki disease. Genome Med 2014; 6 :541.

16 Furukawa S, Matsubara T, Yabuta K. Mononuclear cell subsets and coronary artery lesions in Kawasaki disease. Arch Dis Child 1992;67 :706-8.

17 Matsubara T, Ichiyama T, Furukawa S. Immunological profile of peripheral blood lymphocytes and monocytes/macrophages in Kawasaki disease. Clin Exp Immunol 2005; 141 :381-7.

18 Rivas MN, Lee Y, Wakita D et al. CD8+ T cells contribute to the development of coronary arteritis in the Lactobacillus casei cell wall extract-induced murine model of Kawasaki disease. Arthritis Rheumatol $2017 ; 69: 410-21$.

19 Matsuguma C, Wakiguchi H, Suzuki Y et al. Dynamics of immunocyte activation during intravenous immunoglobulin treatment in Kawasaki disease. Scand J Rheumatol 2019; 48 :491-6.

20 Giordani L, Quaranta MG, Marchesi A et al. Increased frequency of immunoglobulin (Ig)A-secreting cells following Toll-like receptor (TLR)-9 engagement in patients with Kawasaki disease. Clin Exp Immunol $2011 ; 163: 346-53$.

21 Lee HH, Park IH, Shin JS et al. Immunoglobulin $\mathrm{V}(\mathrm{H})$ chain gene analysis of peripheral blood IgMproducing B cells in patients with Kawasaki disease. Yonsei Med J 2009; 50 :493-504.

22 Lee HK, Kim DS, Noh GW et al. Effects of intravenous immune globulin on the peripheral lymphocyte phenotypes in Kawasaki disease. Yonsei Med J 1996; 37 :357-63.

23 Ding Y, Li G, Xiong LJ et al. Profiles of responses of immunological factors to different subtypes of Kawasaki disease. BMC Musculoskelet Disord 2015; 16 :315. 
24 Saulsbury FT. The effect of intravenous immunoglobulin on lymphocyte populations in children with Kawasaki syndrome. Clin Exp Rheumatol 1992;10:617-20.

25 Shingadia D, O'Gorman M, Rowley AH et al. Surface and cytoplasmic immunoglobulin expression in circulating B-lymphocytes in acute Kawasaki disease. Pediatr Res 2001; 50 :538-43.

26 Nie H, Wang S, Wu Q et al. Five immune-gene-signatures participate in the development and pathogenesis of Kawasaki disease. Immun Inflamm Dis 2021; 9 :157-66.

27 Hahn Y, Kim Y, Jo S et al. Reduced frequencies of peripheral interferon-gamma-producing CD4+ and CD4- cells during acute Kawasaki disease. Int Arch Allergy Immunol 2000; 122 :293-8.

28 Burns JC, Hsieh LE, Kumar J et al. Characterization of circulating immune cells in acute Kawasaki disease suggests exposure to different antigens. Clin Exp Immunol 2020; $202: 263-72$.

29 Brogan PA, Shah V, Clarke LA et al. T cell activation profiles in Kawasaki syndrome. Clin Exp Immunol $2008 ; 151: 267-74$.

$30 \mathrm{Ni}$ FF, Li CR, Li Q et al. Regulatory T cell microRNA expression changes in children with acute Kawasaki disease. Clin Exp Immunol 2014; $178: 384-93$.

31 Olivito B, Taddio A, Simonini G et al. Defective FOXP3 expression in patients with acute Kawasaki disease and restoration by intravenous immunoglobulin therapy. Clin Exp Rheumatol 2010;28 :93-7.

32 Jia S, Li C, Wang G et al. The T helper type 17/regulatory $\mathrm{T}$ cell imbalance in patients with acute Kawasaki disease. Clin Exp Immunol 2010; $162: 131-7$.

33 Guo MM, Tseng WN, Ko CH et al. Th17- and Treg-related cytokine and mRNA expression are associated with acute and resolving Kawasaki disease. Allergy 2015; 70 :310-8.

34 Zhou Y, Zhou B, Pache L et al. Metascape provides a biologist-oriented resource for the analysis of systems-level datasets. Nat Commun 2019; $10: 1523$.

35 Pinero J, Bravo A, Queralt-Rosinach N et al. DisGeNET: a comprehensive platform integrating information on human disease-associated genes and variants. Nucleic Acids Res 2017;45 :D833-39.

36 Subramanian A, Tamayo P, Mootha VK et al. Gene set enrichment analysis: a knowledge-based approach for interpreting genome-wide expression profiles. Proc Natl Acad Sci U S A 2005;102 :15545-50.

37 Newman AM, Liu CL, Green MR et al. Robust enumeration of cell subsets from tissue expression profiles. Nat Methods 2015;12:453-7.

38 Miao YR, Zhang Q, Lei Q et al. ImmuCellAI: a unique method for comprehensive T-Cell subsets abundance prediction and its application in cancer immunotherapy. Adv Sci 2020; 7 :1902880.

39 Bindea G, Mlecnik B, Tosolini M et al. Spatiotemporal dynamics of intratumoral immune cells reveal the immune landscape in human cancer. Immunity 2013; 39 :782-95.

40 Galeotti C, Bayry J, Kone-Paut I et al. Kawasaki disease: aetiopathogenesis and therapeutic utility of intravenous immunoglobulin. Autoimmun Rev 2010; 9 :441-8.

41 Rowley AH, Shulman ST. The epidemiology and pathogenesis of kawasaki disease. Front Pediatr 2018; 6 $: 374$.

42 Ishigaki K, Akiyama M, Kanai M et al. Large-scale genome-wide association study in a Japanese population identifies novel susceptibility loci across different diseases. Nat Genet 2020;52 :669-79.

43 Verdoni L, Mazza A, Gervasoni A et al. An outbreak of severe Kawasaki-like disease at the Italian epicentre of the SARS-CoV-2 epidemic: an observational cohort study. Lancet 2020;395:1771-8. 
44 McCrindle BW, Manlhiot C. SARS-CoV-2-related inflammatory multisystem syndrome in children: different or shared etiology and pathophysiology as Kawasaki disease? JAMA 2020; $324: 246-8$.

45 Kumrah R, Vignesh P, Rawat A et al. Immunogenetics of Kawasaki disease. Clin Rev Allergy Immunol 2020; $59: 122-39$.

46 Kuo HC, Pan CT, Huang YH et al. Global investigation of immune repertoire suggests Kawasaki disease has infectious cause. Circ J 2019;83:2070-8.

$47 \mathrm{Xu} \mathrm{M}$, Jiang $\mathrm{Y}$, Wang J et al. Distinct variations of antibody secreting cells and memory B cells during the course of Kawasaki disease. BMC Immunol 2019; 20 :16.

48 Rowley AH, Eckerley CA, Jack HM et al. IgA plasma cells in vascular tissue of patients with Kawasaki syndrome. J Immunol 1997;159 :5946-55.

49 Rowley AH, Shulman ST, Mask CA et al. IgA plasma cell infiltration of proximal respiratory tract, pancreas, kidney, and coronary artery in acute Kawasaki disease. J Infect Dis 2000;182 :1183-91.

50 Rowley AH, Shulman ST, Spike BT et al. Oligoclonal IgA response in the vascular wall in acute Kawasaki disease. J Immunol 2001;166 :1334-43.

51 Chang CJ, Kuo HC, Chang JS et al. Replication and meta-analysis of GWAS identified susceptibility loci in Kawasaki disease confirm the importance of B lymphoid tyrosine kinase (BLK) in disease susceptibility. PLoS One 2013; 8 :e72037.

52 Imayoshi M, Yamamoto S, Watanabe M et al. Expression of CD180, a toll-like receptor homologue, is up-regulated in children with Kawasaki disease. J Mol Med (Berl) 2006; 84 :168-74.

53 Leung DY, Siegel RL, Grady S et al. Immunoregulatory abnormalities in mucocutaneous lymph node syndrome. Clin Immunol Immunopathol 1982; 23 :100-12.

54 Choi IH, Chwae YJ, Shim WS et al. Clonal expansion of CD8+ T cells in Kawasaki disease. J Immunol $1997 ; 159: 481-6$.

55 Kim HS, Noh GW, Kim DS et al. Decreased CD5+ B cells during the acute phase of Kawasaki disease. Yonsei Med J 1996;37:52-8.

56 Furukawa S, Matsubara T, Motohashi T et al. Increased expression of Fc epsilon R2/CD23 on peripheral blood B lymphocytes and serum IgE levels in Kawasaki disease. Int Arch Allergy Appl Immunol 1991; 95 $: 7-12$.

57 Leung DY, Burns JC, Newburger JW et al. Reversal of lymphocyte activation in vivo in the Kawasaki syndrome by intravenous gammaglobulin. J Clin Invest 1987; 79 :468-72.

58 Burns JC, Song Y, Bujold M et al. Immune-monitoring in Kawasaki disease patients treated with infliximab and intravenous immunoglobulin. Clin Exp Immunol 2013; 174 :337-44.

$59 \mathrm{Xu}$ M, Jiang Y, Wang $\mathrm{J}$ et al. Distribution of distinct subsets of circulating $\mathrm{T}$ follicular helper cells in Kawasaki disease. BMC Pediatr 2019; 19 :43.

60 Ehara H, Kiyohara K, Izumisawa Y et al. Early activation does not translate into effector differentiation of peripheral CD8T cells during the acute phase of Kawasaki disease. Cell Immunol 2010;265 :57-64.

61 Ikeda K, Yamaguchi K, Tanaka T et al. Unique activation status of peripheral blood mononuclear cells at acute phase of Kawasaki disease. Clin Exp Immunol 2010; 160 :246-55.

62 Popper SJ, Shimizu C, Shike H et al. Gene-expression patterns reveal underlying biological processes in Kawasaki disease. Genome Biol 2007; 8 :R261.

63 Ge X, Li CR, Yang J et al. Aberrantly decreased levels of NKG2D expression in children with kawasaki disease. Scand J Immunol 2013; 77 :389-97. 
64 Furukawa S, Matsubara T, Tsuji K et al. Comparison of Kawasaki disease and infectious mononucleosis in terms of natural killer cell and CD8+ T cell subsets. J Infect Dis 1991; 163:416-7.

65 Furukawa S, Matsubara T, Jujoh K et al. Peripheral blood monocyte/macrophages and serum tumor necrosis factor in Kawasaki disease. Clin Immunol Immunopathol 1988; $48: 247-51$.

66 Katayama K, Matsubara T, Fujiwara M et al. CD14+CD16+ monocyte subpopulation in Kawasaki disease. Clin Exp Immunol 2000;121:566-70.

67 Koga M, Ishihara T, Takahashi M et al. Activation of peripheral blood monocytes and macrophages in Kawasaki disease: ultrastructural and immunocytochemical investigation. Pathol Int 1998;48 :512-7.

68 Ariga S, Koga M, Takahashi M et al. Maturation of macrophages from peripheral blood monocytes in Kawasaki disease: immunocytochemical and immunoelectron microscopic study. Pathol Int 2001;51 :257-63.

69 Abe J, Matsuda A. Biomarkers associated with unresponsiveness to IVIG in children with Kawasaki disease. Nihon Rinsho Meneki Gakkai Kaishi 2013; 36 :27-34.

70 Wright VJ, Herberg JA, Kaforou M et al. Diagnosis of Kawasaki disease using a minimal whole-blood gene expression signature. JAMA Pediatr 2018; 172 :e182293.

71 Popper SJ, Watson VE, Shimizu C et al. Gene transcript abundance profiles distinguish Kawasaki disease from adenovirus infection. J Infect Dis 2009; 200 :657-66.

72 Amano S, Hazama F, Kubagawa H et al. General pathology of Kawasaki disease. On the morphological alterations corresponding to the clinical manifestations. Acta Pathol Jpn 1980; 30 :681-94.

73 Hamada H, Suzuki H, Abe J et al. Inflammatory cytokine profiles during Cyclosporin treatment for immunoglobulin-resistant Kawasaki disease. Cytokine 2012; 60 :681-5.

74 Tremoulet AH, Pancoast P, Franco A et al. Calcineurin inhibitor treatment of intravenous immunoglobulin-resistant Kawasaki disease. J Pediatr 2012; 161 :506-12.e1.

75 Lindquist ME, Hicar MD. B cells and antibodies in Kawasaki disease. Int J Mol Sci 2019; 20 :1834.

76 Hwang JY, Lee KY, Rhim JW et al. Assessment of intravenous immunoglobulin non-responders in Kawasaki disease. Arch Dis Child 2011;96 :1088-90.

77 Qian W, Tang Y, Yan W et al. A comparison of efficacy of six prediction models for intravenous immunoglobulin resistance in Kawasaki disease. Ital J Pediatr 2018; 44 :33.

78 Cho HJ, Bak SY, Kim SY et al. High neutrophil: lymphocyte ratio is associated with refractory Kawasaki disease. Pediatr Int 2017;59:669-74.

$79 \mathrm{Wu}$ G, Yue P, Ma F et al. Neutrophil-to-lymphocyte ratio as a biomarker for predicting the intravenous immunoglobulin-resistant Kawasaki disease. Medicine (Baltimore) 2020; 99 :e18535.

80 Gamez-Gonzalez LB, Hamada H, Cisneros Castolo M et al. Vital signs as predictor factors of intravenous immunoglobulin resistance in patients with Kawasaki disease. Clin Pediatr (Phila) 2018;57 :1148-53.

81 Wang Y, Li Z, Hu G et al. Unique molecular patterns uncovered in kawasaki disease patients with elevated serum gamma glutamyl transferase levels: implications for intravenous immunoglobulin responsiveness. PLoS One 2016; 11 :e0167434.

82 Ye Q, Gong FQ, Shang SQ et al. Intravenous immunoglobulin treatment responsiveness depends on the degree of CD8+ T cell activation in Kawasaki disease. Clin Immunol 2016; $171: 25-31$.

83 Li Y, Ren P, Dawson A et al. Single-cell transcriptome analysis reveals dynamic cell populations and differential gene expression patterns in control and aneurysmal human aortic tissue. Circulation 2020; 142 $: 1374-88$. 
84 Luo W, Wang Y, Zhang L et al. Critical role of cytosolic DNA and its sensing adaptor STING in Aortic degeneration, dissection, and rupture. Circulation 2020; $141: 42-66$.

Tables

Table 1. Basic information of the KD datasets

\begin{tabular}{llcr}
\hline Datasets & Type and number & Sample source & Year \\
\hline GSE9863 & Acute KD $(\mathrm{n}=20)$ and convalescent KD $(\mathrm{n}=20)$ & Whole blood & 2007 \\
GSE9864 & Acute KD $(\mathrm{n}=53)$ & Whole blood & 2007 \\
GSE18606 & Normal $(\mathrm{n}=9)$, acute KD $(\mathrm{n}=20)$, and convalescent KD $(\mathrm{n}=19)$ & Whole blood & 2009 \\
GSE63881 & Acute KD $(\mathrm{n}=171)$ and convalescent KD $(\mathrm{n}=170)$ & Whole blood & 2014 \\
GSE68004 & Normal $(\mathrm{n}=37)$, cKD $(\mathrm{n}=76)$, inKD $(\mathrm{n}=13), \mathrm{HAdV}(\mathrm{n}=19)$, and GAS $(\mathrm{n}=14)$ & Whole blood & 2016 \\
GSE73461 & Normal $(\mathrm{n}=55), \mathrm{KD}(\mathrm{n}=77), \mathrm{DB}(\mathrm{n}=52), \mathrm{DV}(\mathrm{n}=94)$, and ID $(\mathrm{n}=84)$ & Whole blood & 2018 \\
GSE73463 & Acute KD $(\mathrm{n}=146)$ and convalescent KD $(\mathrm{n}=87)$ & Whole blood & 2018 \\
GSE64486 & Normal $(\mathrm{n}=7)$ and KD $(\mathrm{n}=8)$ & Coronary artery & 2015 \\
\hline
\end{tabular}

Normal controls in GSE64486 had normal coronary artery histology. Abbreviations: KD, Kawasaki disease; cKD, complete KD; inKD, incomplete KD; HadV, adenovirus; GAS, group A streptococcal disease; DB, definite bacterial infection; DV, definite viral infection; ID, inflammatory diseases.

Table 2. Comparison of peripheral blood immune cell abundances between normal controls, acute KD samples and febrile controls (ImmuCellAI)

\begin{tabular}{|c|c|c|c|c|c|c|c|c|c|c|}
\hline \multirow[t]{2}{*}{$\begin{array}{l}\text { Immune } \\
\text { Cells }\end{array}$} & \multicolumn{6}{|c|}{ GSE68004GSE68004GSE68004GSE68004GSE68004GSE68004 } & \multicolumn{4}{|c|}{ GSE73461GSE73461GSE73461GSE73461 } \\
\hline & $\begin{array}{l}\text { Normal } \\
(\mathrm{n}=37)\end{array}$ & $\begin{array}{l}\mathrm{cKD} \\
(\mathrm{n}=76)\end{array}$ & $\begin{array}{l}\operatorname{inKD} \\
(n=13)\end{array}$ & $\begin{array}{l}\mathrm{HadV} \\
(\mathrm{n}=19)\end{array}$ & $\begin{array}{l}\text { GAS } \\
(\mathrm{n}=14)\end{array}$ & $p$ value & $\begin{array}{l}\text { Normal } \\
(\mathrm{n}=55)\end{array}$ & $\begin{array}{l}\mathrm{KD} \\
(\mathrm{n}=77)\end{array}$ & $\begin{array}{l}\mathrm{DB} \\
(\mathrm{n}=52)\end{array}$ & $\begin{array}{l}\text { DV } \\
(\mathrm{n}=94)\end{array}$ \\
\hline $\begin{array}{l}\text { B } \\
\text { cell }\end{array}$ & 0.185 & 0.151 & 0.139 & 0.147 & 0.159 & 0.035 & 0.226 & 0.170 & 0.179 & 0.204 \\
\hline $\begin{array}{l}\mathrm{CD} 4 \\
\mathrm{~T}\end{array}$ & 0.160 & 0.113 & 0.108 & 0.142 & 0.162 & $<0.001$ & 0.211 & 0.166 & 0.170 & 0.190 \\
\hline $\begin{array}{l}\text { CD8 } \\
\mathrm{T}\end{array}$ & 0.336 & 0.186 & 0.198 & 0.300 & 0.238 & $<0.001$ & 0.384 & 0.274 & 0.268 & 0.331 \\
\hline $\begin{array}{l}\mathrm{CD} 4 \\
\text { naive }\end{array}$ & 0.041 & 0.007 & 0.011 & 0.026 & 0.013 & $<0.001$ & 0.109 & 0.074 & 0.094 & 0.095 \\
\hline iTreg & 0.097 & 0.099 & 0.079 & 0.073 & 0.073 & 0.389 & 0.174 & 0.134 & 0.138 & 0.168 \\
\hline nTreg & 0.084 & 0.071 & 0.061 & 0.076 & 0.069 & 0.287 & 0.300 & 0.299 & 0.284 & 0.286 \\
\hline $\operatorname{Tr} 1$ & 0.210 & 0.180 & 0.153 & 0.239 & 0.203 & 0.022 & 0.082 & 0.083 & 0.063 & 0.075 \\
\hline Th1 & 0.220 & 0.140 & 0.139 & 0.183 & 0.169 & $<0.001$ & 0.191 & 0.147 & 0.178 & 0.191 \\
\hline Th2 & 0.178 & 0.193 & 0.128 & 0.230 & 0.223 & 0.060 & 0.140 & 0.173 & 0.189 & 0.174 \\
\hline Th17 & 0.169 & 0.351 & 0.395 & 0.196 & 0.237 & $<0.001$ & 0.417 & 0.394 & 0.417 & 0.432 \\
\hline Tfh & 0.078 & 0.040 & 0.064 & 0.065 & 0.070 & $<0.001$ & 0.056 & 0.043 & 0.034 & 0.044 \\
\hline Tcm & 0.226 & 0.124 & 0.120 & 0.215 & 0.167 & $<0.001$ & 0.182 & 0.150 & 0.141 & 0.186 \\
\hline Tem & 0.018 & 0.010 & 0.005 & 0.012 & 0.014 & 0.037 & 0.101 & 0.108 & 0.107 & 0.107 \\
\hline $\begin{array}{l}\mathrm{CD} 8 \\
\text { naive }\end{array}$ & 0.205 & 0.142 & 0.097 & 0.190 & 0.153 & $<0.001$ & 0.164 & 0.114 & 0.122 & 0.123 \\
\hline $\begin{array}{l}\text { Cytoxic } \\
\mathrm{T}\end{array}$ & 0.020 & 0.023 & 0.037 & 0.039 & 0.033 & 0.032 & 0.162 & 0.193 & 0.196 & 0.193 \\
\hline $\begin{array}{l}\text { Exhaustec } \\
\mathrm{T}\end{array}$ & $\mathrm{d} 0.028$ & 0.034 & 0.052 & 0.029 & 0.037 & 0.723 & 0.003 & 0.004 & 0.013 & 0.004 \\
\hline
\end{tabular}




\begin{tabular}{|c|c|c|c|c|c|c|c|c|c|c|}
\hline $\begin{array}{l}\text { Immune } \\
\text { Cells }\end{array}$ & GSE68 & 4GSE6 & 4GSE6 & 4GSE6 & 4GSE6 & 4GSE68004 & GSE7: & 1GSE7 & 1GSE7: & 1GSE73461 \\
\hline MAIT & 0.157 & 0.129 & 0.121 & 0.144 & 0.138 & 0.236 & 0.227 & 0.212 & 0.201 & 0.222 \\
\hline $\begin{array}{l}\text { Gamma } \\
\text { delta } \\
\mathrm{T}\end{array}$ & 0.152 & 0.072 & 0.083 & 0.144 & 0.134 & $<0.001$ & 0.284 & 0.235 & 0.212 & 0.258 \\
\hline NKT & 0.083 & 0.170 & 0.157 & 0.142 & 0.146 & $<0.001$ & 0.155 & 0.204 & 0.169 & 0.165 \\
\hline NK & 0.292 & 0.228 & 0.200 & 0.262 & 0.239 & $<0.001$ & 0.287 & 0.239 & 0.248 & 0.250 \\
\hline $\begin{array}{l}\text { Dendritic } \\
\text { cell }\end{array}$ & 0.498 & 0.556 & 0.507 & 0.499 & 0.515 & 0.308 & 0.318 & 0.359 & 0.326 & 0.356 \\
\hline Monocyte & 0.154 & 0.238 & 0.261 & 0.229 & 0.209 & $<0.001$ & 0.259 & 0.370 & 0.365 & 0.338 \\
\hline Macropha & agel15 & 0.148 & 0.166 & 0.122 & 0.136 & 0.008 & 0.208 & 0.264 & 0.253 & 0.198 \\
\hline Neutrophi & iiD.118 & 0.251 & 0.281 & 0.150 & 0.195 & $<0.001$ & 0.245 & 0.385 & 0.360 & 0.298 \\
\hline Infiltratior & mosedste & 0.748 & 0.745 & 0.741 & 0.742 & 0.941 & 0.748 & 0.779 & 0.759 & 0.757 \\
\hline
\end{tabular}

The above immune cell abundances are average values. Abbreviations: KD, Kawasaki disease; cKD, complete $\mathrm{KD}$; inKD, incomplete KD; HadV, adenovirus; GAS, group A streptococcal disease; DB, definite bacterial infection; DV, definite viral infection; ID, inflammatory diseases.

Table 3. Changes in peripheral blood immune cells in KD

\begin{tabular}{|c|c|c|c|}
\hline Immune cells & Reported data & References & References \\
\hline B cells & $\begin{array}{l}* \text { The number and } \\
\text { relative percentage of B } \\
\text { lymphocytes in the } \\
\text { peripheral blood of acute } \\
\text { KD patients is increased. } \\
\text { * After IVIG, the } \\
\text { percentage of B cells is } \\
\text { decreased. * In patients } \\
\text { with acute KD, } \\
\text { circulating IgA B } \\
\text { lymphocytes and plasma } \\
\text { cells are reduced. }{ }^{*} \text { There } \\
\text { are decreased CD } 5+\text { B } \\
\text { cells in acute KD. } * \text { In } \\
\text { acute KD samples, the } \\
\text { absolute count of CD23+ } \\
\text { B lymphocytes and the } \\
\text { ratio of CD23+ B } \\
\text { lymphocytes to B } \\
\text { lymphocytes are high } \\
\text { compared to those in } \\
\text { convalescent KD samples } \\
\text { and control subjects. }\end{array}$ & $\begin{array}{l}{[12,16,17,20-23,46-53]} \\
{[17,20,22,24][25,26,54]} \\
{[22,55][56]}\end{array}$ & $\begin{array}{l}{[12,16,17,20-23,46-53]} \\
{[17,20,22,24][25,26,54]} \\
{[22,55][56]}\end{array}$ \\
\hline
\end{tabular}




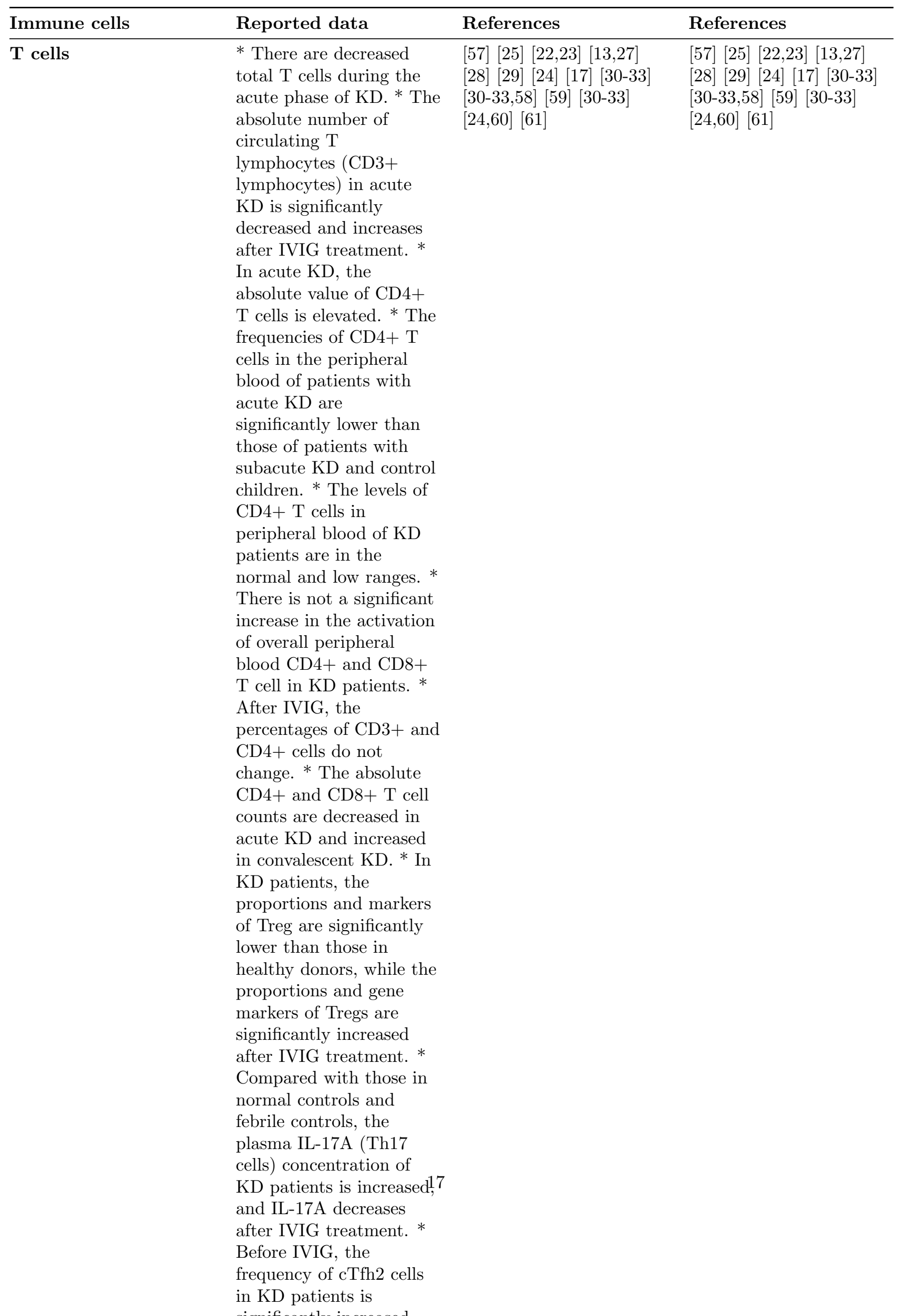




\begin{tabular}{|c|c|c|c|}
\hline Immune cells & Reported data & References & References \\
\hline NK cells & $\begin{array}{l}* \text { The absolute number } \\
\text { of NK cells in the acute } \\
\text { phase of KD is } \\
\text { significantly decreased } \\
\text { and increases after } \\
\text { IVIG treatment. }\end{array}$ & {$[20,23,25,62-64]$} & {$[20,23,25,62-64]$} \\
\hline 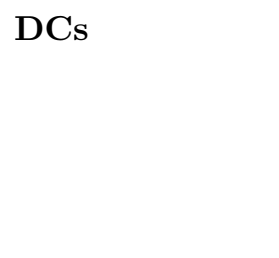 & $\begin{array}{l}* \text { Myeloid DC }(\mathrm{mDC}) \\
\text { cells are increased in } \\
\text { the peripheral blood of } \\
\text { patients with acute KD } \\
\text { and decline in the } \\
\text { subacute phase. }\end{array}$ & {$[58]$} & {$[58]$} \\
\hline $\begin{array}{l}\text { Monocytes } \\
\text { \&Macrophages }\end{array}$ & $\begin{array}{l}\text { * In acute KD, the } \\
\text { numbers of peripheral } \\
\text { blood CD14+ mono- } \\
\text { cytes/macrophages and } \\
\text { activated } \\
\text { CD14+CD23+ mono- } \\
\text { cytes/macrophages are } \\
\text { increased. }{ }^{*} \text { The } \\
\text { number of CD14+ } \\
\text { CD16+ (FcrRIII) } \\
\text { mono- } \\
\text { cytes/macrophages in } \\
\text { acute KD is increased } \\
\text { and decreases with } \\
\text { IVIG treatment. } \\
\text { Immunocytochemical } \\
\text { and immunoelectron } \\
\text { microscopic studies } \\
\text { have shown that } \\
\text { monocytes partly } \\
\text { differentiate into } \\
\text { macrophages in acute } \\
\text { KD peripheral } \\
\text { circulation. }\end{array}$ & {$[16,65][17,66][67,68]$} & {$[16,65][17,66][67,68]$} \\
\hline
\end{tabular}




\begin{tabular}{|c|c|c|c|}
\hline Immune cells & Reported data & References & References \\
\hline Neutrophils & $\begin{array}{l}\text { * Neutrophils are } \\
\text { significantly increased in } \\
\text { acute KD and decreased } \\
\text { in convalescent KD. } * \\
\text { Compared with that in } \\
\text { febrile controls, the } \\
\text { number of neutrophils in } \\
\text { patients with acute KD is } \\
\text { increased, and these } \\
\text { neutrophils are also } \\
\text { activated, as evidenced } \\
\text { by their expression of } \\
\text { various late-stage } \\
\text { granulocyte-specific } \\
\text { genes. * Compared with } \\
\text { patients with acute } \\
\text { adenovirus infection or } \\
\text { systemic adverse } \\
\text { reactions, KD patients } \\
\text { have higher expression } \\
\text { levels of neutrophil } \\
\text { activation-related genes, } \\
\text { but the levels are not } \\
\text { higher than those of } \\
\text { scarlet fever patients. }\end{array}$ & {$[12-15][69,70][71]$} & \\
\hline
\end{tabular}

\section{Figure Legends}

Figure 1. The inflammatory response is the most significant hallmark of Kawasaki disease (KD). (A) Verified differentially expressed genes (VDEGs) in the KD datasets GSE18606, GSE68004, and GSE73461. (B) Top 20 Metascape enrichment analysis results of VDEGs. (C) Metascape enrichment network showing the similarity of enrichment terms within and between the top 20 clusters. (D) Gene set enrichment analysis (GSEA) of the top 10 significant hallmarks in KD samples.

Figure 2. Atlas of circulating immune cells in acute and convalescent KD. Proportions of 22 types of immune cells in (A) normal controls, (B) acute KD samples, and (C) convalescent KD samples based on CIBERSORT. (D) Changes in the proportions of innate immune cells and adaptive immune cells during the progression and regression of KD. (E) Map showing the differences in KD immune cell proportions (CIBERSORT). The former three columns represent the comparisons of immune cell proportions between acute KD samples and normal controls. The latter three columns represent the comparisons of immune cell proportions between acute KD and convalescent KD samples. (F) Map showing the differences in KD immune cell abundances (ImmuCellAI). The former three columns represent the comparisons of immune cell abundances between the acute KD group and the normal group. The latter 3 columns represent the comparisons of immune cell abundances between acute KD and convalescent KD samples. The superscripts in (E) and (F) are the names of the datasets, and the color of the square indicates the significance. The deeper red the color is, the higher the abundance or proportion of the immune cell is in acute KD samples compared with normal controls or convalescent KD samples. The blue represents the opposite pattern. NA, not applicable; NS, not significant.

Figure 3. The relationship between the response of KD patients to intravenous immunoglobulin (IVIG) and the levels of peripheral blood immune cells. (A) Sankey diagram of the relationship between IVIG 
response and aneurysm formation in GSE63881. (B) The former two columns represent the comparisons of immune cell proportions (CIBERSORT) between IVIG responders and IVIG nonresponders in acute KD. The latter two columns represent the comparisons of immune cell proportions between IVIG responders and IVIG nonresponders in convalescent KD. (C) The former two columns represent the comparisons of immune cell abundances (ImmuCellAI) between IVIG responders and IVIG nonresponders in acute KD. The latter two columns represent the comparisons of immune cell abundances between IVIG responders and IVIG nonresponders in convalescent KD. (D) The former three columns represent the comparisons of immune cell abundances (immune cell markers) between IVIG responders and IVIG nonresponders in acute KD. The latter two columns represent the comparisons of immune cell abundances between IVIG responders and IVIG nonresponders in convalescent KD. The superscripts are the names of the datasets, and the color of the square indicates the significance. The deeper red the color is, the higher the abundance or proportion of the immune cell is in IVIG responders compared with IVIG nonresponders. The blue represents the opposite pattern. NA, not applicable; NS, not significant.

Figure 4. Comparison of immune cell abundances in coronary arteries between normal controls and KD samples (ImmuCellAI).

Figure 5. Schematic diagram of KD pathogenesis. The high-incidence period of KD is between 6 months and 5 years of age, which coincides with the immune trough period when maternal immunity declines and the child's own immune system is immature. Nonspecific pathogens infect genetically susceptible children through the respiratory tract, and the most harmful effect of the systemic immune response induced by these pathogens is coronary vasculitis. Necrotizing arteritis occurs in the first two weeks of KD and is manifested by neutrophils infiltrating the coronary arteries. This effect is followed by inflammatory infiltration of monocytes, macrophages, B cells, CD4 $+\mathrm{T}$ cells, and CD8 $+\mathrm{T}$ cells during the period of subacute chronic arteritis. However, in acute KD, adaptive immune cells such as B cells, CD4+ T cells and CD8+ T cells in the peripheral blood are significantly decreased, which suggests that adaptive immune cells are selectively recruited from the peripheral circulation to KD target tissues.

\section{Supplementary Information}

Table S1. Comparison of peripheral blood immune cell abundances between normal controls and acute KD samples (ImmuCellAI).

Table S2. Comparison of peripheral blood immune cell abundances between acute KD and convalescent KD samples (ImmuCellAI).

Figure S1. Summary of the enrichment analysis in DisGeNET.

Figure S2. Comparison of immune cell proportions (CIBERSORT) between acute KD samples and normal controls in (A) GSE18606, (B) GSE68004, and (C) GSE73461.

Figure S3. Comparison of immune cell proportions (CIBERSORT) between acute KD and convalescent KD samples in (A) GSE18606, (B) GSE63881, and (C) GSE73463.

Figure S4. Comparison of immune cell abundances (ImmuCellAI) between acute KD samples and normal controls in (A) GSE18606, (B) GSE68004, and (C) GSE73461.

Figure S5. Comparison of immune cell abundances (ImmuCellAI) between acute KD and convalescent KD samples in (A) GSE9863, (B) GSE18606, (C) GSE63881, and (D) GSE73463.

Figure S6. Map of the differences in KD immune cell abundances (as determined based on immune cell markers). The former three columns represent the comparisons of immune cell abundances between acute KD samples and normal controls. The latter 4 columns represent the comparisons of immune cell abundances between acute $\mathrm{KD}$ and convalescent $\mathrm{KD}$ samples.

Figure S7. Comparison of immune cell abundances (determined based on immune cell markers) between acute KD samples and normal controls in (A) GSE18606, (B) GSE68004, and (C) GSE73461. 
Figure S8. Comparison of immune cell abundances (determined based on immune cell markers) between acute KD and convalescent KD samples in (A) GSE9863, (B) GSE18606, (C) GSE63881, and (D) GSE73463.

Figure S9. The decrease in CD4 $+\mathrm{T}$ cells and CD $8+\mathrm{T}$ cells and the increase in neutrophils in KD peripheral blood are specific. (A) Differences in the proportions of CD8+ T cells and neutrophils between the normal controls, KD samples and febrile controls in GSE68004. (B) Differences in the proportions of CD8+ T cells and neutrophils between the normal controls, KD samples and febrile controls in GSE73461. (C) Differences in the abundances of cytotoxic T cells (quantified with marker CD8A), T helper cells (CD4) and neutrophils (CXCR2) between the normal controls, KD samples and febrile controls in GSE68004. (D) Differences in the abundances of cytotoxic $\mathrm{T}$ cells, $\mathrm{T}$ helper cells and neutrophils between the normal controls, KD samples and febrile controls in GSE73461. Abbreviations: cKD, complete KD; InKD: incomplete KD; AdV: adenovirus; GAS: group A Streptococcus. ${ }^{*} \mathrm{p}<0.05,{ }^{* *} \mathrm{p}<0.01,{ }^{* * *} \mathrm{p}<0.001$, ns: not significant.

Figure S10. The abundance of neutrophils in peripheral blood can be used as a biomarker for KD diagnosis. (A) The ROC curve of differential diagnosis between KD samples and normal controls, HAdV samples, and GAS samples in GSE68004. (B) The ROC curve of differential diagnosis between KD samples and normal controls, DB samples, DV samples and ID samples in GSE73461. Abbreviations: KD, Kawasaki disease; cKD, complete KD; inKD, incomplete KD; HadV, adenovirus; GAS, group A Streptococcus; DB, definite bacterial infection; DV, definite viral infection; ID, inflammatory disease; ROC, receiver operating characteristic; AUC, area under the ROC curve.

Figure S11. Comparison of immune cell proportions (CIBERSORT) between IVIG responders and IVIG nonresponders in (A) GSE18606 acute KD, (B) GSE63881 acute KD, (C) GSE18606 convalescent KD, and (D) GSE63881 convalescent KD samples.

Figure S12. Comparison of immune cell abundances (ImmuCellAI) between IVIG responders and IVIG nonresponders in (A) GSE18606 acute KD, (B) GSE63881 acute KD, (C) GSE18606 convalescent KD, and (D) GSE63881 convalescent KD samples.

Figure S13. Comparison of immune cell abundances (immune cell markers) between IVIG responders and IVIG nonresponders in (A) GSE9864 acute KD, (B) GSE18606 acute KD, (C) GSE63881 acute KD, (D) GSE18606 convalescent KD, and (E) GSE63881 convalescent KD samples.

Figure S14. The relationship between aneurysms and the level of peripheral blood immune cells in KD. (A) Comparison of immune cell proportions (determined based on CIBERSORT) between KD patients with coronary artery aneurysm, dilated coronary artery and normal coronary artery. (B) Comparison of immune cell abundances (determined based on ImmuCellAI) between KD patients with coronary artery aneurysm, dilated coronary artery and normal coronary artery. (C) Comparison of immune cell abundances (determined based on immune cell markers) between KD patients with coronary artery aneurysm, dilated coronary artery and normal coronary artery.

Figure S15. Comparison of the immune cell abundances in coronary arteries between normal controls and KD samples (determined based on immune cell markers). ${ }^{*} \mathrm{p}<0.05$, ${ }^{* *} \mathrm{p}<0.01,{ }^{* * *} \mathrm{p}<0.001$, ns: not significant 
A

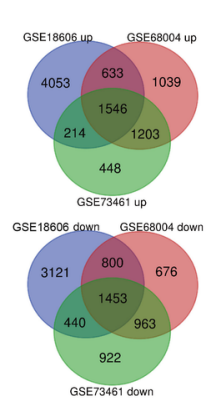

B

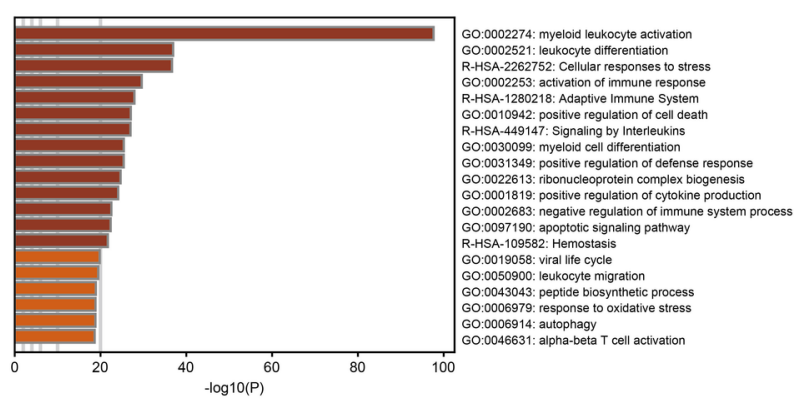

D

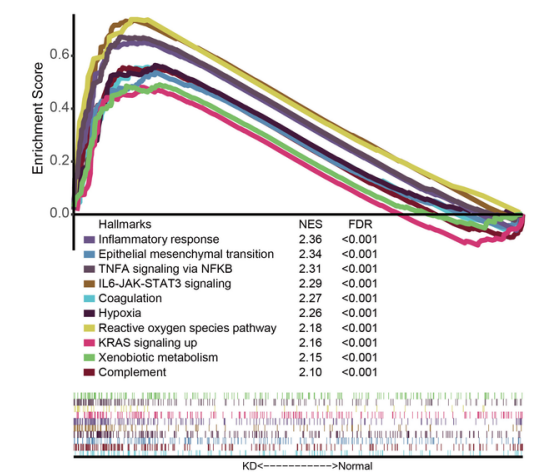


A

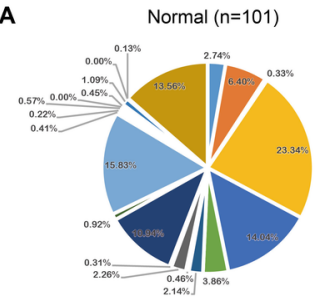

- B cells naive

- T cells CD4

- Monocytes

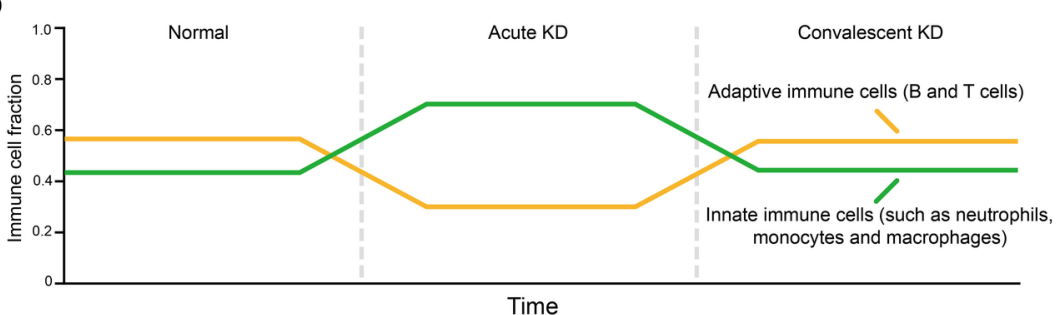

E

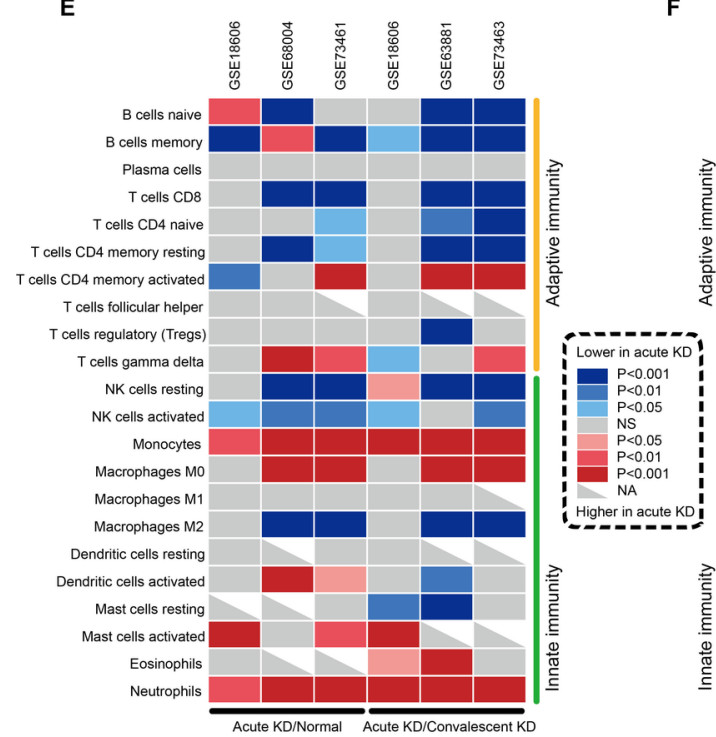

C Convalescent KD $(n=272)$

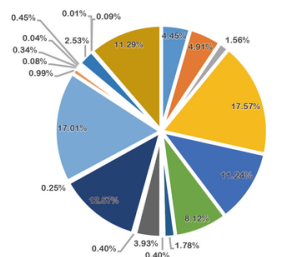

- T cells CD4 naive $\quad-T$ cells CD4 memory resting

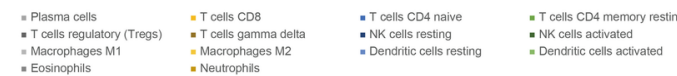
- Eosinophis

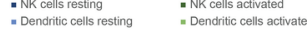

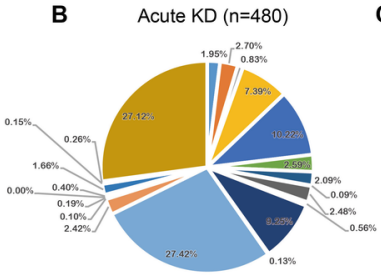

immune cells
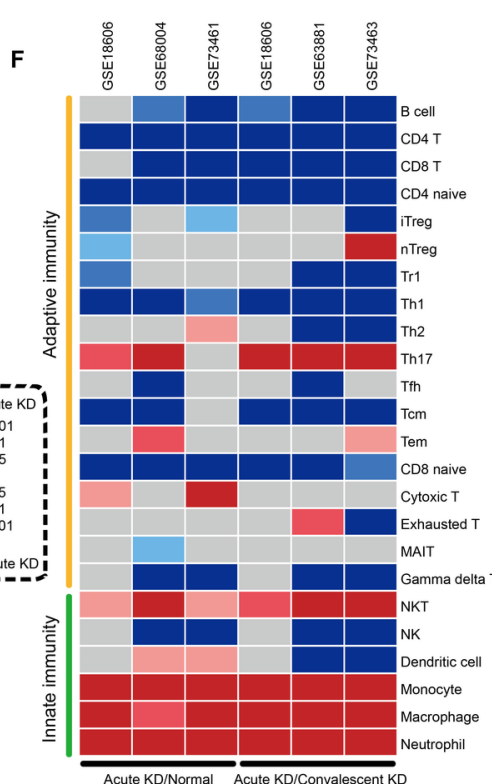
A

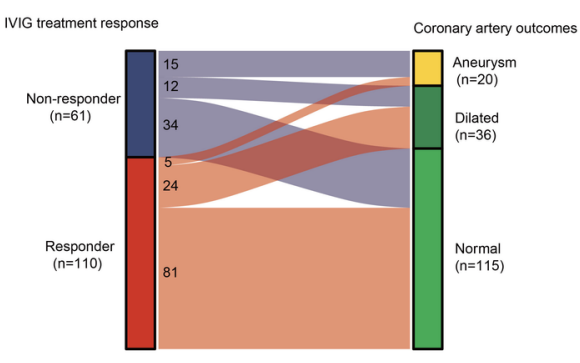

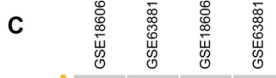

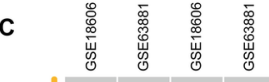

B
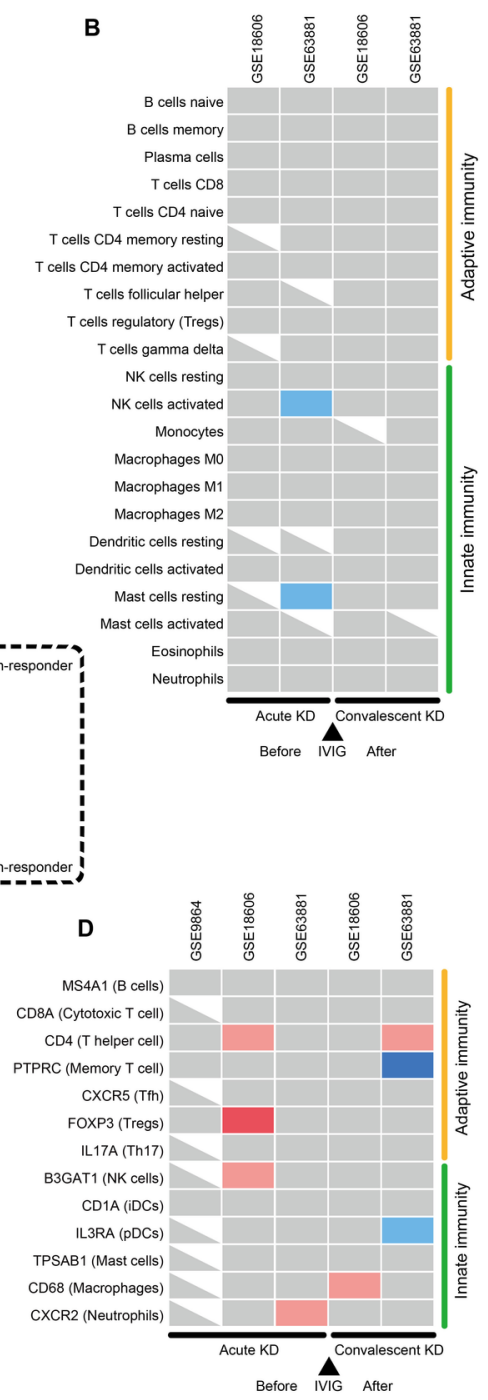

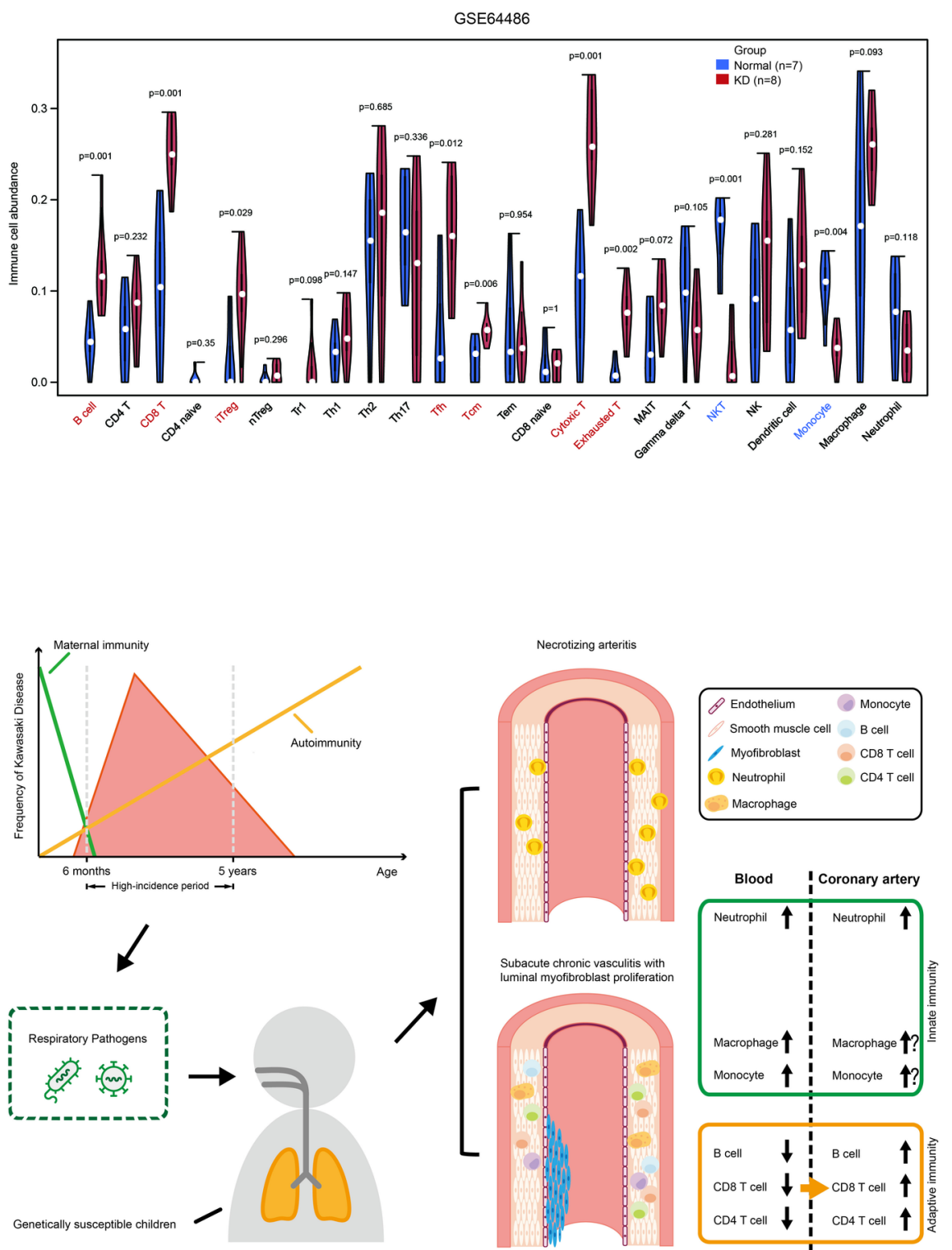